\title{
A Migrating Retained Intracranial Bullet Presenting with Hydrocephalus and Alterations of Consciousness: A Case Report and
} Literature Review

\author{
Mohammad Sadegh Masoudi, ${ }^{1,2,}{ }^{*}$ Fariborz Ghafarpasand, ${ }^{3}$ and Shahaboddin Yousefi ${ }^{4}$ \\ ${ }^{1}$ Assistant Professor of Neurosurgery, Trauma Research Center of AJA University of Medical Sciences, Tehran, Iran \\ ${ }^{2}$ Neurosurgery Department of Shiraz University of Medical Sciences, Shiraz, Iran \\ ${ }^{3}$ Resident of Neurosurgery, Namazi Hospital, Shiraz University of Medical Sciences, Shiraz, Iran \\ ${ }^{4}$ Resident of Neurosurgery, Shiraz University of Medical Sciences, Shiraz, Iran \\ "Corresponding author: Mohammad Sadegh Masoudi, Assistant Professor of Neurosurgery, Trauma Research Center of AJA University of Medical Sciences, Tehran, Iran. E-mail: \\ masoudims@gmail.com
}

Received 2017 June 13; Accepted 2017 June 16.

\begin{abstract}
Migrating intracranial bullets are a dilemma to neurosurgeons, as their management is challenging, and the removal process is associated with high mortality and morbidity rates. They are also associated with alterations in consciousness and focal neurological defects. We herein report a 40-year-old man with a retained intracranial AK-47 bullet for 22 years, admitted to our emergency department with decreased level of consciousness. He was diagnosed with meningitis and obstructive hydrocephalus, given the pressure effect of the bullet over the aqueduct of Sylvius. He underwent placement of external ventricular drainage (EVD) and received broad-spectrum antibiotics. However, he passed away due to complications of meningitis. Postmortem examination revealed that the bullet had entered the anterior perforated substance from ethmoidal air cells and migrated to the brain ventricular system in 22 years. Accordingly, conservative management of intracranial retained bullets is recommended.
\end{abstract}

Keywords: Intracranial Bullet, Gunshot Wound, Migrating Bullet, Hydrocephalus, Decreased Level of Consciousness

\section{Introduction}

Migration of retained intracranial metallic bullets and missile fragments is a complication of penetrating gunshot wounds (GSW), reported in $4.2 \%$ of all surviving civilians with GSWs (1). There are several reports in the literature regarding the migrating retained intracranial bullets (1-4). All these reports indicate a tendency for intracranial bullets to migrate through the ventricular system, cisternae, and cerebral hemispheres (5-8).

There are several hypotheses explaining the mechanism of migration of a retained metallic fragment within brain tissues. Higher gravidity of the bullet in comparison to the brain tissue, pulsatile force of the brain and cerebrospinal fluid (CSF), and softening of the brain tissue through fragment trajectory are among the most common mechanisms (9). The presence of a bullet or metal missile fragment as a foreign body is associated with several complications, such as meningitis, abscess formation, CSF fistula, epilepsy, hematoma, hydrocephalus, altered level of consciousness, and confusion (2, 3, 5, 10, 11).

We herein report a migrating intracranial bullet, retained for 22 years, which had entered through ethmoidal air cells, migrated through the ventricles, and ended in the posterior part of the third ventricle, causing hydro- cephalus and meningitis.

\section{Case Presentation}

A 40-year-old Afghan man presented to the emergency room with decreased level of consciousness and fever since several days ago. He had no medical history and was a warrior during the Afghan civil war (1995, 22 years ago). He remembered having a wound on his upper lip during a battle for which he did not receive any medical treatment, except wound care. He also reported several episodes of behavioral changes and speech problems without any evaluation. He migrated to Iran after 2 years, and by the age of 33 years, he had a relatively good health condition. He developed several episodes of generalized tonic-clonic convulsions for which he did not seek any medical assistance.

He was admitted to our center 3 years ago due to status epilepticus. Neuroimaging revealed a bullet in the calvarium (Figures 1 and 2). He was discharged with anticonvulsants and was not admitted to our center until 2017. He was in a good condition, and seizures were managed by anticonvulsants until 2017 when he suddenly presented to the hospital with decreased level of consciousness.

His Glasgow Coma scale (GCS) score on admission was 7 (M, 5; V, 1; E, 1), and both pupils were reactive to light. The 


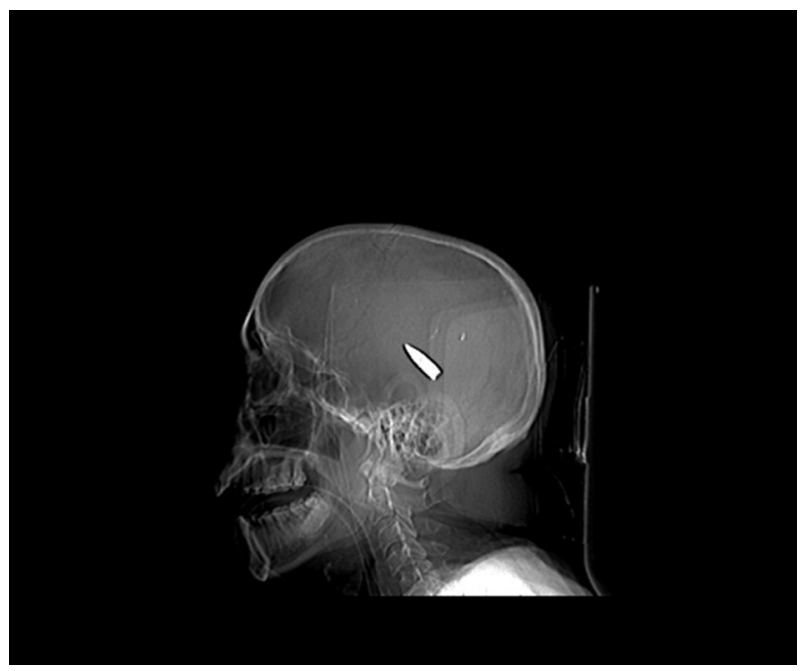

Figure 1. The Lateral Skull View of the Patient with an Intracranial Bullet

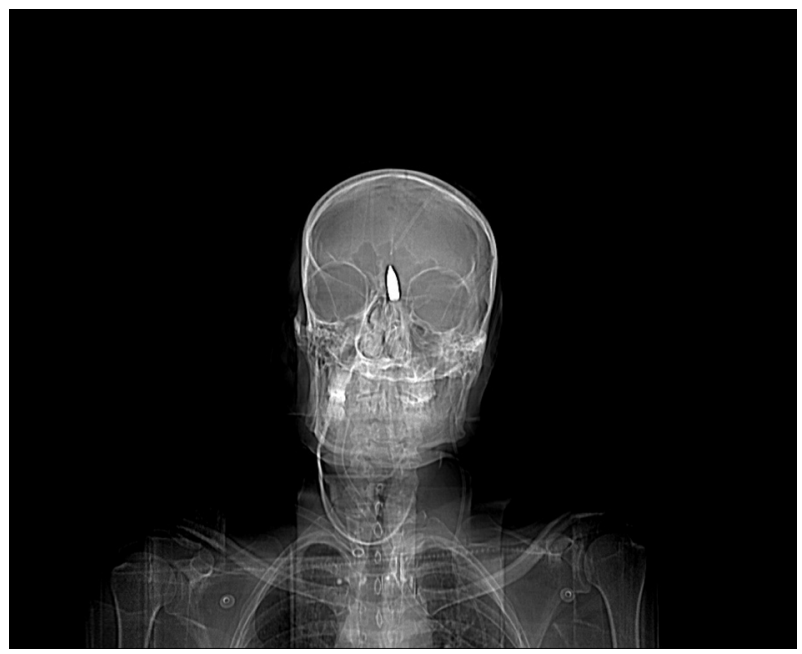

Figure 2. The Anteroposterior View of the Patient with a Bullet in the Midline Position

brain CT scan revealed acute hydrocephalus and an AK-47 bullet in the ventricular system (Figure 3). An external ventricular drainage (EVD) was inserted, and meningitis was diagnosed for which broad-spectrum antibiotics were administered. After the CSF was cleared and the antibiotic course was finished, a ventriculoperitoneal shunt was inserted and the patient received conservative therapy.

The patient passed away on the 28th day of admission due to complications of meningitis, including pneumonia and CSF fistula. The 3-dimensional skull CT scan and postmortem study revealed the entrance of the bullet to the posterior ethmoidal air cells and the anterior perforating

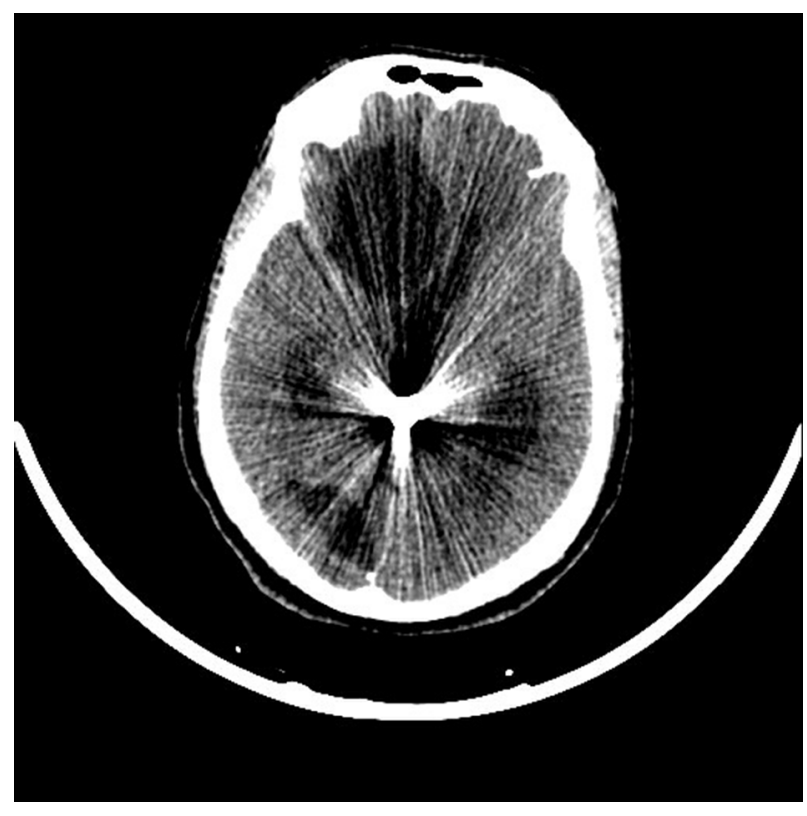

Figure 3. The CT Scan of the Patient with Hydrocephalus and a Retained Bullet in the Ventricular System

substance (Figure 4). The bullet had migrated through the lateral ventricles and finally to the pineal region and the third ventricle (Figure 5).

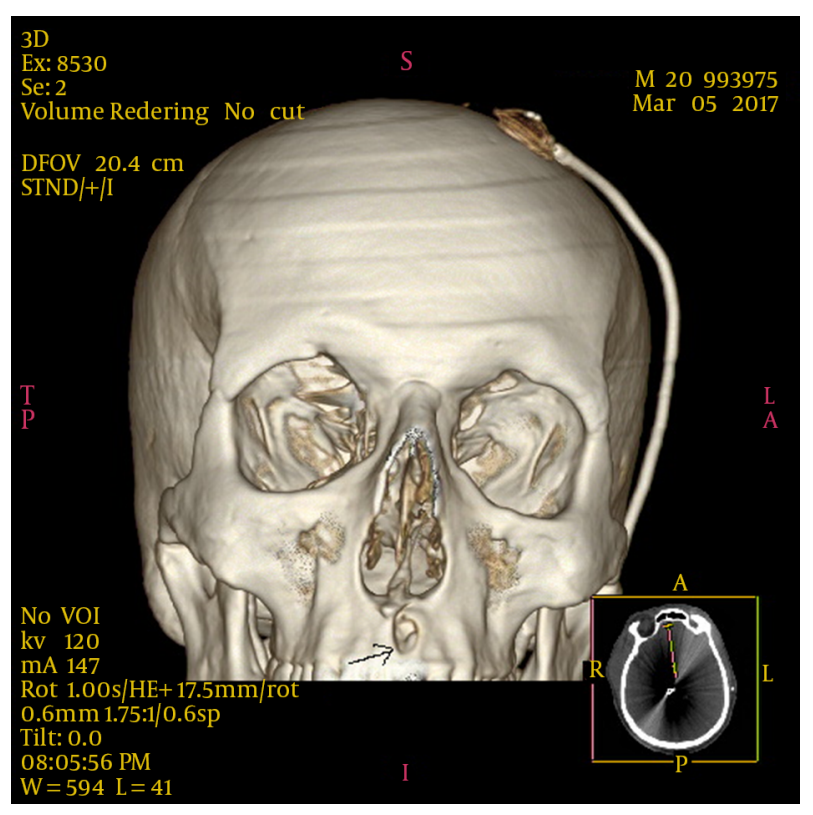

Figure 4. The 3D CT Scan of the Skull and Face Revealing the Site of Bullet Entrance to the Maxilla 


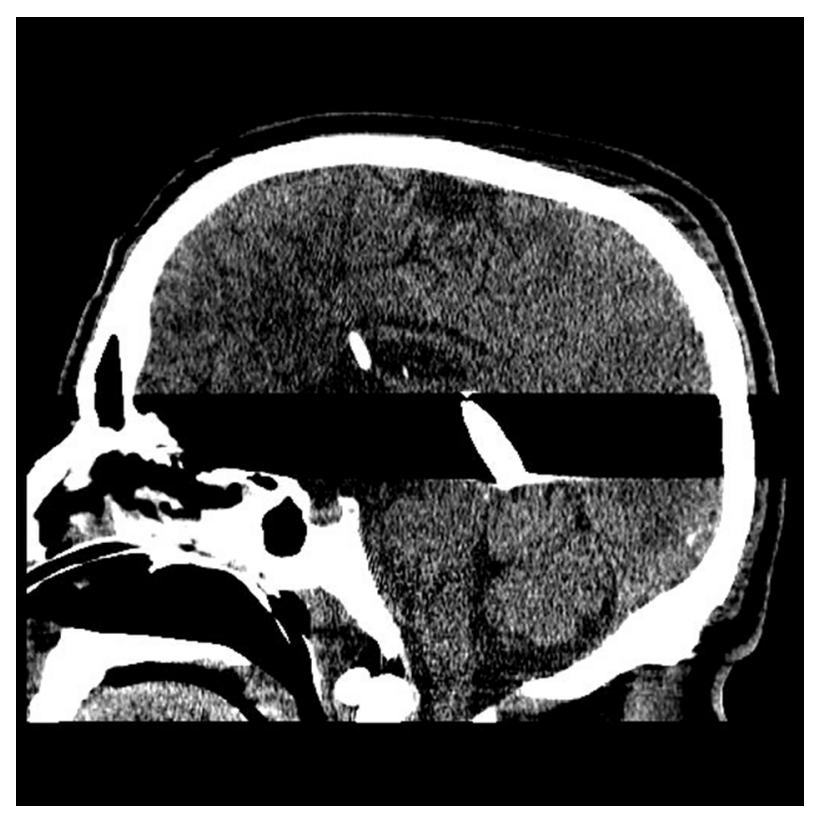

Figure 5. The Sagittal View of Brain CT Scan Showing Bullet Entrapment in the Posterior Region of the Third Ventricle with Compression on the Pineal Region and Aqueduct of Sylvius Causing Hydrocephalus

\section{Discussion}

The civilian GSWs have been studied in different settings and countries with different incidence, outcome, and management outlines $(12,13)$. Although migration of an intracranial projectile is an uncommon condition, severity of complications associated with such events often contributes to poor prognostic implications $(1,14)$. Penetrating brain injury from a high-velocity projectile creates a complex pattern of permanent tissue damage. The bullet track left in the wake of the missile creates a potential nidus for foreign body migration, local brain necrosis, and external contamination of devitalized wound tissues (15).

Regarding penetrating GSWs, reports have described projectiles moving above, below, and through a defect in the tentorium cerebelli, passing through the tentorial incisure, and moving inside the spinal canal and thecal sac. Overall, intact bullets, copper jackets, lead cores, and pellets can migrate through the body. Rapp et al. (1) reviewed 213 neurosurgical patients with GSWs of the head and estimated that approximately $4.2 \%$ of the bullet fragments migrated; the earliest migration of a bullet fragment was discovered 36 hours post injury. Moreover, other authors have found that $<0.1 \%$ to $10 \%$ of bullets migrate inside the head $(2,3,11)$.

In comparison with the study by Rapp et al. the bullet in this case migrated within approximately 10.5 hours af- ter the shooting (1). There is a tendency for foreign bodies to migrate into or out of the ventricles, within the cisternae, in the ipsilateral cerebral hemisphere, or within the cerebellum $(7,8)$. Migration may occur under a variety of clinical conditions, the most significant of which is migration through the ventricular system, subarachnoid space, necrotic liquefied brain tissue, and along the trajectory.

In conclusion, delayed presentation of intracranial retained bullets is relatively uncommon in patients with penetrating brain injuries. Migration of retained bullets is a delayed presentation. Bullets in the ventricular system have a great possibility of migration, and ventriculitis and hydrocephalus may develop.

\section{References}

1. Rapp LG, Arce CA, McKenzie R, Darmody WR, Guyot DR, Michael DB. Incidence of intracranial bullet fragment migration. Neurol Res. 1999;21(5):475-80. [PubMed: 10439428].

2. Chute DJ, Newman K, Bready RJ, Benjamin ED. Case Report of a Migrating Bullet: An Unusual Cause of Postmortem Confusion.J Forensic Sci. 2017 doi: 10.1111/1556-4029.13428. [PubMed: 28217834].

3. Esene IN, Ashour AM, Mohamed OY, Mahmoud WS, Fahkr A. Migrating Intraventricular Gunshot Pellet: A Case Report. J Clin Case Rep. 2015;05(07) doi: 10.4172/2165-7920.1000564.

4. Rammo RA, DeFazio MV, Bullock MR. Management of migrating intracranial bullets: lessons learned from surviving an AK-47 bullet through the lateral brainstem. World Neurosurg. 2012;77(3-4):591 e1924. doi:10.1016/j.wneu.2011.06.015. [PubMed: 22120363].

5. Karabagli H. Spontaneous movement of bullets in the interhemispheric region. Pediatr Neurosurg. 2005;41(3):148-50. doi: 10.1159/000085873. [PubMed: 15995333].

6. Kerin DS, Fox R, Mehringer CM, Grinnell V, Miller RE, Hieshima GB. Spontaneous migration of a bullet in the central nervous system. Surg Neurol. 1983;20(4):301-4. [PubMed: 6623340].

7. Milhorat TH, Elowitz EH, Johnson RW, Miller JI. Spontaneous movement of bullets in the brain. Neurosurgery. 1993;32(1):140-3. [PubMed: 8421545].

8. Ozkan U, Ozates M, Kemaloglu S, Guzel A. Spontaneous migration of a bullet into the brain. Clin Neurol Neurosurg. 2006;108(6):573-5. doi: 10.1016/j.clineuro.2005.03.002. [PubMed:16905434].

9. Salvati M, Cervoni L, Rocchi G, Rastelli E, Delfini R. Spontaneous movement of metallic foreign bodies. Case report. J Neurosurg Sci. 1997;41(4):423-5. [PubMed: 9555653].

10. Gressot LV, Chamoun RB, Patel AJ, Valadka AB, Suki D, Robertson $\mathrm{CS}$, et al. Predictors of outcome in civilians with gunshot wounds to the head upon presentation. J Neurosurg. 2014;121(3):645-52. doi: 10.3171/2014.5.JNS131872. [PubMed: 24995781].

11. Joseph B, Aziz H, Pandit V, Kulvatunyou N, O’Keeffe T, Wynne J, et al. Improving survival rates after civilian gunshot wounds to the brain. $J$ Am Coll Surg. 2014;218(1):58-65. doi: 10.1016/j.jamcollsurg.2013.08.018. [PubMed: 24055384].

12. DeCuypere M, Muhlbauer MS, Boop FA, Klimo PJ. Pediatric intracranial gunshot wounds: the Memphis experience. J Neurosurg Pediatr. 2016;17(5):595-601. doi:10.3171/2015.7.PEDS15285. [PubMed: 26728100].

13. Solmaz I, Kural C, Temiz C, Secer HI, Duz B, Gonul E, et al. Traumatic brain injury due to gunshot wounds: a single institution's experience with 442 consecutive patients. Turk Neurosurg. 2009;19(3):216-23. [PubMed: 19621284]. 
14. Rinaldi A, Gazzeri R, Conti L, Schiavone M, Cava M, Tirelli GC. Cranioorbital missile wound and bullet migration. Case report. J Neurosurg Sci. 2000;44(2):107-12. [PubMed: 11105841].

15. Aarabi B, Tofighi B, Kufera JA, Hadley J, Ahn ES, Cooper C, et al. Pre- dictors of outcome in civilian gunshot wounds to the head. $J$ Neurosurg. 2014;120(5):1138-46. doi: 10.3171/2014.1.JNS131869. [PubMed: 24506239]. 